\title{
Dural arteriovenous fistulas of the cavernous sinus - clinical case and treatment
}

\author{
A. Chiriac, N. Dobrin ${ }^{\star}$, St.M. Iencean, I. Poeata \\ "Grigore T. Popa" University of Medicine and Pharmacy, Iasi \\ "Prof. Dr. N. Oblu” Clinic Emergency Hospital, Iasi*
}

\begin{abstract}
The purpose of our article is to present the results of our treatment of dural arteriovenous fistula of the cavernous sinus by glue embolization of the external carotid artery feeders. By this case presentation we try to clarify the clinical course, with the dural carotid cavernous fistula (CCF), characterizing a pallet of symptoms, paying special attention to radiological finding and endovascular treatment.
\end{abstract}

Dural arteriovenous fistulas represent $10 \%$ to $15 \%$ of all intracranial arteriovenous malformations (A. Fox, G. Duckwiler, "Dural Arteriovenous Fistula," presented at the annual meeting of the American Society of Neuroradiology, St Louis, Mo, June 1992). Dural arteriovenous fistulas are rare clinical situation, especially examples involving the cavernous sinus. Most dural fistulas are acquired conditions, typically occurring in postmenopausal women, but sometimes in other patients in association with other condition $[1,3]$. These dural fistulas are most often "spontaneous" cavernous carotid shunts (usually low-flow) $[2,4,5]$, usually related to a past trauma or surgery. The classical triad, represented by pulsating exophthalmos, conjunctival chemosis, and pulsatile-tinnitus are well-known clinical symptoms of these lesions but are not necessary present in the majority of the patients as first indicators.

The anatomy of these vascular malformations consists of multiple arterial feeders flowing into cavernous sinus. The arterial feeders are usually meningeal branches arising from the internal carotid artery (ICA) or the external carotid artery (ECA).

However, there are few reports of large series [1], and the clinical entity is not widely known. The purpose of this paper is to present a clinical case of a patient with dural cavernous sinus fistulae, clarify the clinical symptoms course and special attention to results of endovascular treatment.

\section{Case report}

A 42-year-old man presented with a 3month history of pulsatile headaches, tinnitus and hyperaemia of left eye. He had a one month history of blurred vision and diplopia in all gaze positions. He declares a history of a head trauma with occipital impact three years ago. The patient was otherwise healthy and was a nonsmoker. 


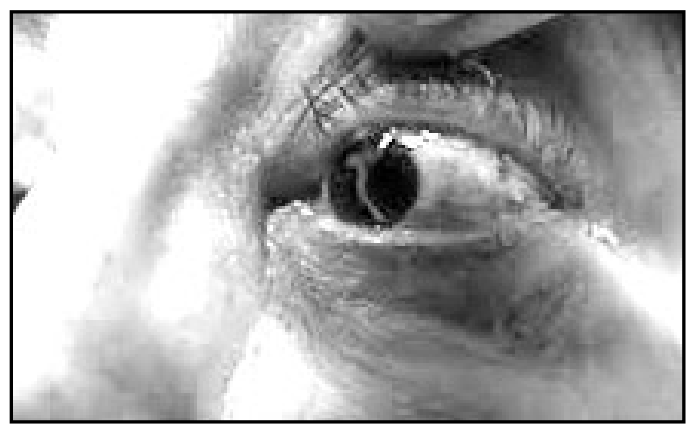

Figure 1 - Patient photo with affected eye

Ophthalmological examination revealed conjuctival chemosis with proptosis and arterialisation of the conjuctival blood vessels. Patient presents exophthalmia but with no anisocoria. Retinal blot haemorrhages and bilateral optic nerve cupping were noted at fundal examination. Visual acuity was 20/50 in the right eye and 20/30 in the left eye.

The complete neurological and physical examination revealed no other aspects.

The patient was investigated by craniocerebral magnetic resonance imaging (MRI) and magnetic resonance angiography (arterial TOF), performed at an outside institution.

These images showed an enlarged left superior ophthalmic vein. Cerebral angiography demonstrated a Barrow Type $\mathrm{C}$ indirect CCF supplied by an internal maxillary branches of the left ECA.

Drainage from the cavernous sinus was via superior ophthalmic veins, angular vein and anterior facial vein. There was no cortical venous drainage or drainage to the inferior petrosal sinus from the cavernous sinus.

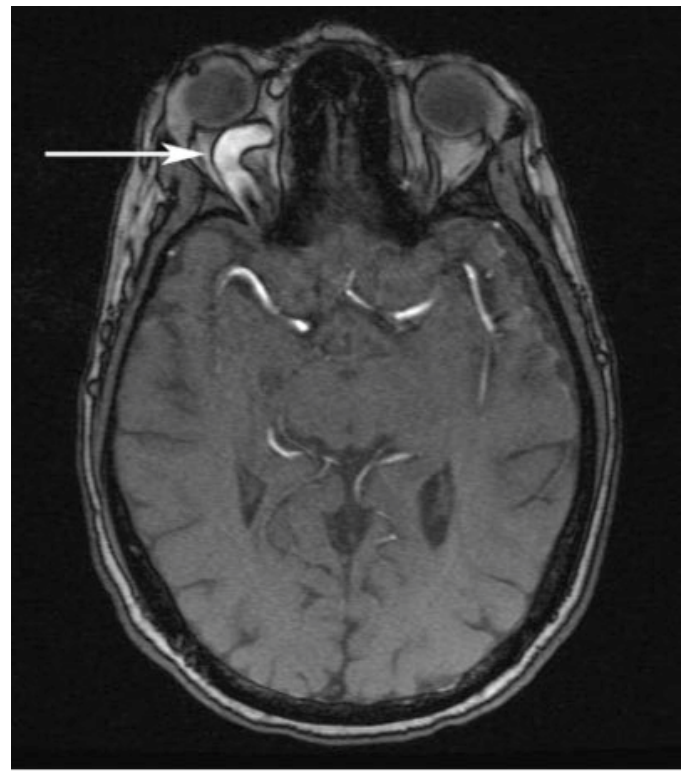

Figure 2 - MRI show an enlarged left superior ophthalmic vein

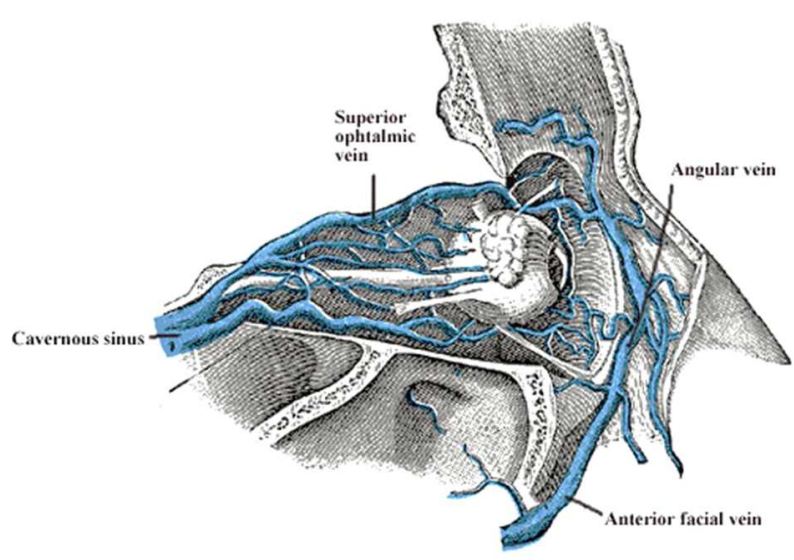

Figure 3 - Anatomy of cranio-facial vein [5] 
DOI: 10.2478/romneu-2014-0018
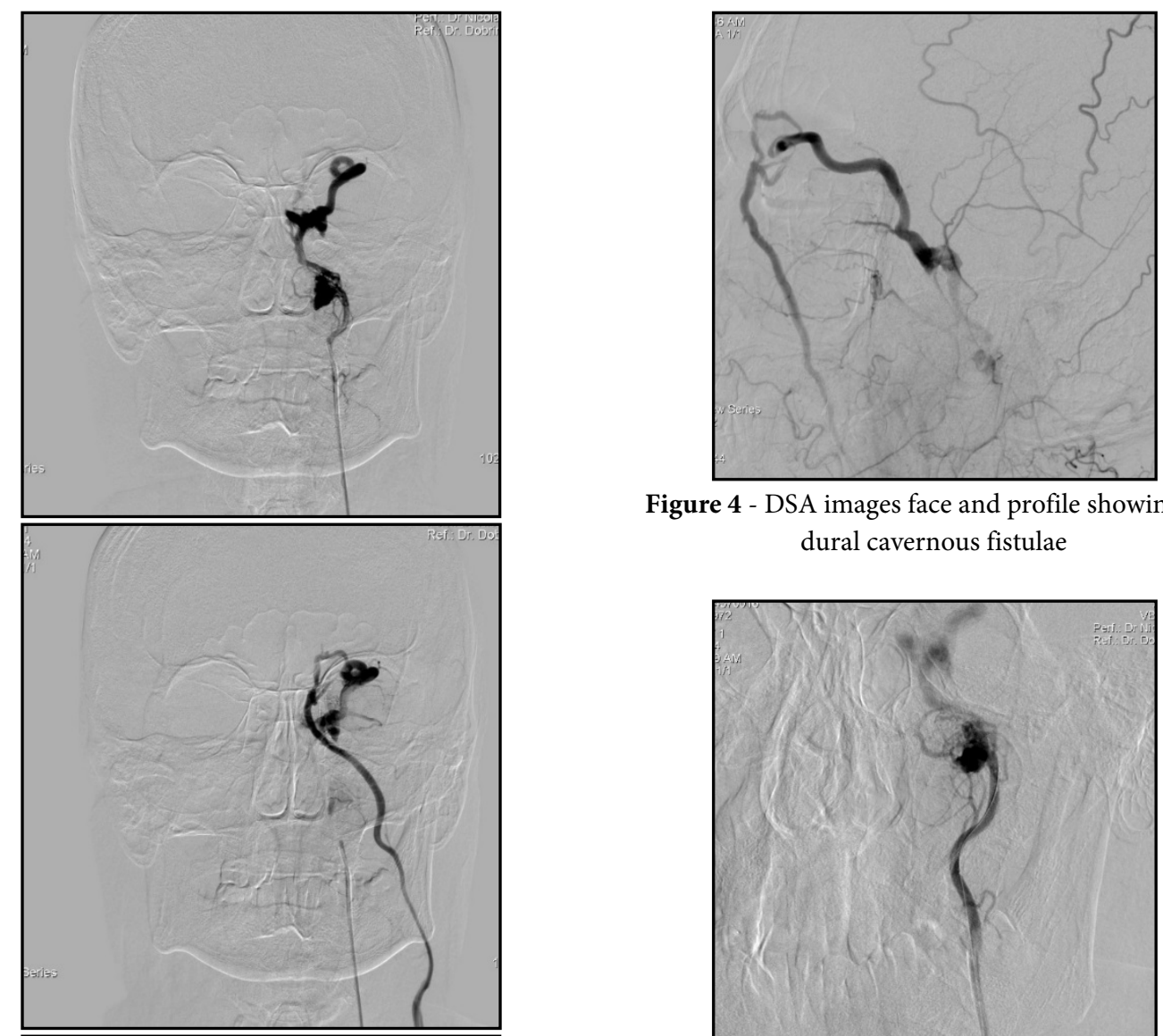

Figure 4 - DSA images face and profile showing the dural cavernous fistulae
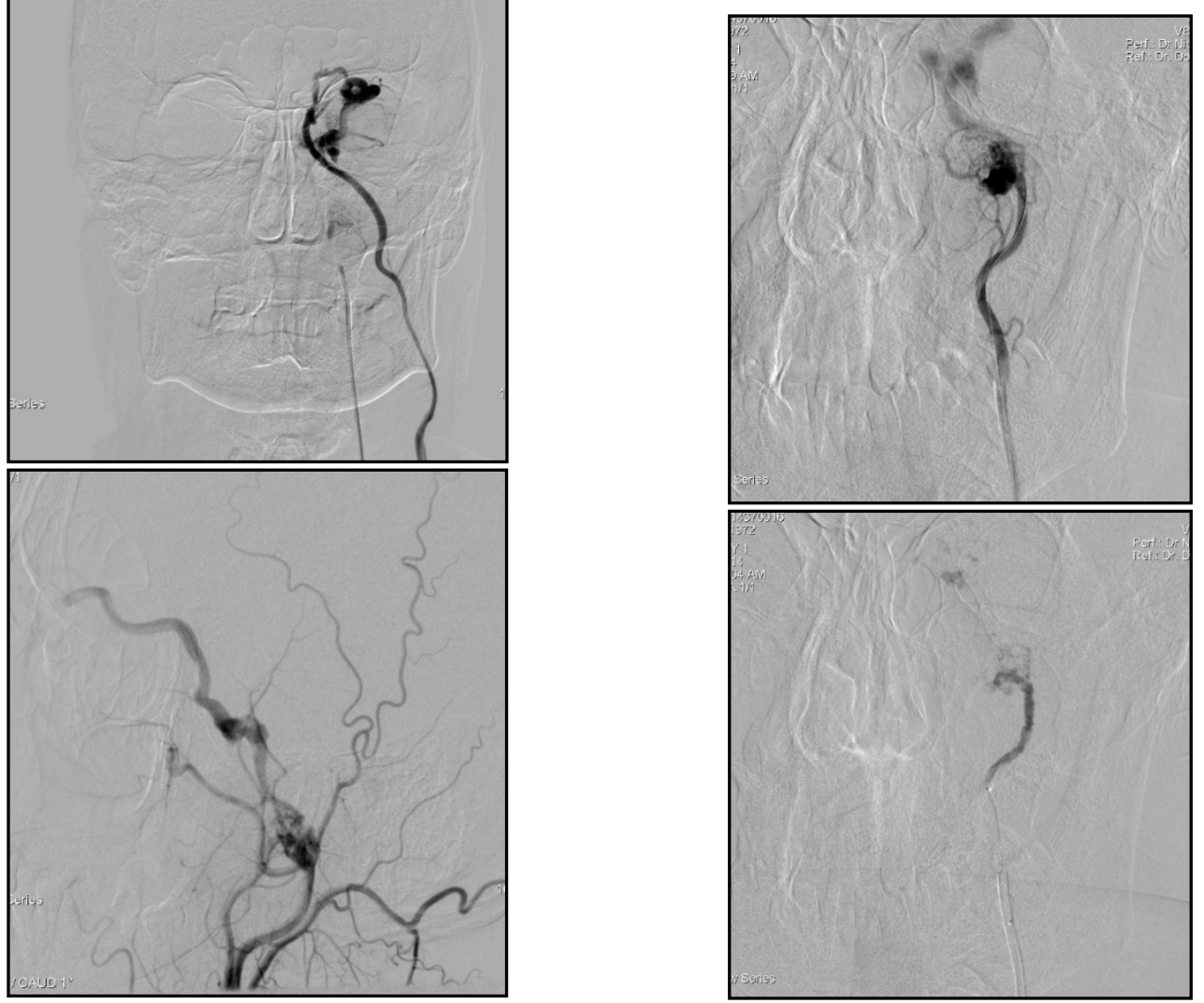

Figure 5 - DSA images showing arterial pedicle microcatheterization and glue injection 
Transarterial embolisation via the external carotid arterial feeder was used as it was feared that this might promote a complete occlusion of the fistula. A mixture of $2 \mathrm{ml}$ of lipiodol and GlueBran 2 was used to complete occlusion of the dural fistula. A complete remission of symptoms was achieved in 48 hours postintervention.
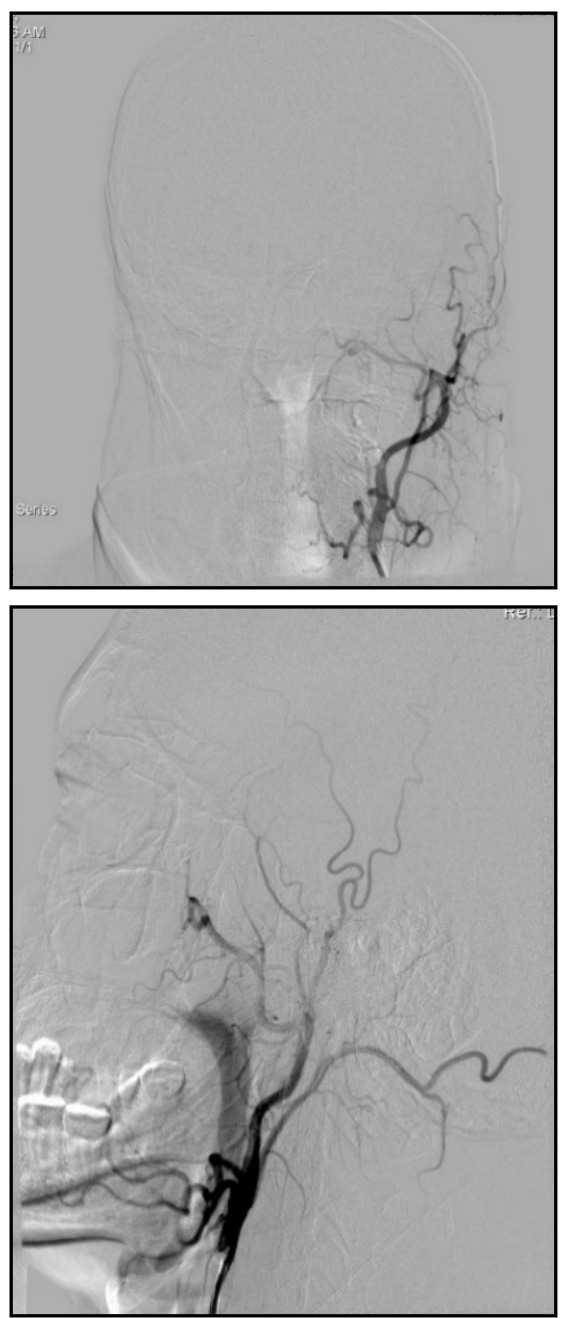

Figure 6 - DSA images showing total dural cavernous fistulae acclusion

\section{Discussion}

A correct diagnosis of a dural cavernous sinus fistula is clinically difficult and may take long time. Usually, there are low-flow fistulas with no severe symptoms as in traumatic highflow cavernous sinus fistulas. Most patients do not have bruit or pulsatile exophthalmos, which indicate a vascular lesion. However, diagnosis should always be considered in patients with a red eye and arterialized episcleral vessels [1].

Classical triad clinical symptoms represented by conjunctival chemosis, pulsating exophthalmos and bruit, are well known to be a strong indication of carotid cavernous fistula related to venous drainage routes. Total or partial absence of this classical triad, made patients often have been initially misdiagnosed. Taniguchi et al. [1,7] showed that absence of a bruit was the principal cause of misdiagnosis, while Walker and Allegre $[1,3]$ reported the same situation for exophthalmos.

Akira Kurata et al., presented evidence that dural CCF patients usually do not show the classical triad, cranial nerve palsies being the most common initial symptoms in their patient series. Usually, multiple venous drainages route may explain the limitation of symptoms to only cranial nerve palsies. This appears due to dispersion of the arterial flow which will conceal disguise the classical triad. Also, in only $36 \%$ of cases at least one of the classical triad was the initial symptom.

Unilateral head pain was reported by Newton and al.[1] to be the frequently early symptom usually interpreted as an unusual 
DOI: 10.2478/romneu-2014-0018

migraine attack. They also described, unilateral headache as mostly transient symptom with duration of less than 1 month.

Imaging diagnosis can be performed by contrast-enhanced CT and MR imaging, MR angiography, or Doppler imaging studies, which will show enlarged draining veins. MR imaging (T1-weighted images) may suggest thrombosis or slow flow in the superior ophthalmic vein when this structure appears hyperintense (instead of the usual flow void) $[1,2]$. Phase-contrast MR angiography and Doppler studies permit identification of flow reversal in the enlarged superior ophthalmic vein.

The majority of patients with dural arteriovenouse fistulas of the cavernous sinus follow an extremely benign clinical course. Serious complications such as intracerebral hemorrhage are very rare. Exceptionally, some patients may present decrease of visual acuity including central retinal thrombosis and cortical venous reflux evident on angiography. Spontaneous regression in dural CCF is not uncommon phenomenon and was noted in 5 of 11 cases reported by Newton and Hoyt, 19 of 26 patients by Sasaki et al. [10], and three of 18 by Vinuela et al $[1,2]$.

The two major endovascular approaches for dural arteriovenouse fistulas of the cavernous sinus treatment are represented by transarterial embolization and transvenouse route embolization. Recently, transvenouse embolization has been proposed as a more appropriate curative treatment than transarterial embolization. Anyway, this may result in serious difficult outcomes like embolic stroke as reported by Halbach et al.
$[1,2]$, especially if performed without prior arterial flow reduction by transarterial embolization.

Yamashita et al. reported complication occurring in 7 of their 16 patients undergoing transvenouse embolization. They described an epidural extravasation due to perforation of the inferior petrosal sinus, and the other 6 transient aggravations of symptoms (chemosis and sixth/third cranial nerve palsy in three each) $[1,2]$.

Cortical venous reflux was demonstrated to be an important feature associated with major complications. En extravasation from the uncal vein during a transvenouse embolization for superior ophthalmic vein and inferior ophthalmic vein via the inferior petrosal sinus was reported by Araki et al. He show the importance of early obliterating of cortical venous drainage as soon as possible, even when the reflux is small $[1,2]$.

\section{Conclusions}

Endovascular embolization of dural arteriovenous fistulas of the cavernous sinus via external carotid artery feeders has a high success rate. In some fistulas, the endovascular occlusion of external carotid artery system is sufficient, whereas it will cause the decrease of symptoms. In bilateral fistulas situation, endovascular embolization of the most symptomatic side may be followed by a spontaneous occlusion of the contralateral fistula. In case of insufficient fistula occlusion by external carotid artery feeders embolization or a predominant fistula supply from internal carotid artery, a venous embolization is indicated. The aim of venous route 
embolization is to occlude the fistula without redirecting venous drainage to cortical structures.

\section{Correspondence:}

Stefan Mircea Iencean

mirceasteffan@yahoo.com

\section{References}

1. D. Quinones, G. Duckwiler, P. Y. Gobin, R. A. Goldberg, and F. Vinuela: Embolization of Dural Cavernous Fistulas via Superior Ophthalmic Vein Approach, AJNR Am J Neuroradiol, May 1997, 18:921928;

2. Akira Kurata, Sachio Suzuki, Kazuhisa Iwamoto, Kuniaki Nakahara, Makoto Sasaki, Chihiro Kijima, Madoka Inukai, Katsutoshi Abe, Jun Niki, Kimitoshi Satou, Kiyotaka Fujii, Shinichi Kan. Dural arteriovenous fistulas in the cavernous sinus: clinical research and treatment. ISRN neurology, 2011;
3. Pero, Guglielmo, et al. Onyx embolization of dural arteriovenous fistulas of the cavernous sinus through the superior pharyngeal branch of the ascending pharyngeal artery. Journal of neurointerventional surgery, 2014, neurintsurg-2013-011067. rep.;

4. Barrow, D. L., Spector, R. H., Braun, I. F., Landman, J. A., Tindall, S. C., \& Tindall, G. T. (1985). Classification and treatment of spontaneous carotid-cavernous sinus fistulas. Journal of neurosurgery, 62(2), 248-256.

5. Nelson, P. K., Russell, S. M., Woo, H. H., Alastra, A. J., \& Vidovich, D. V. (2003). Use of a wedged microcatheter for curative transarterial embolization of complex intracranial dural arteriovenous fistulas: indications, endovascular technique, and outcome in 21 patients. Journal of neurosurgery, 98(3), 498-506.

6. VanLandingham, M., Fox, B., Hoit, D., Elijovich, L., \& Arthur, A. S. (2014). Endovascular Treatment of Intracranial Dural Arteriovenous Fistulas. Neurosurgery, 74, S42-S49.

7. Macdonald, A., Plaha, P., \& Byrne, J. (2014). An unusual presentation of a dural arteriovenous fistula of the sphenoparietal sinus. Journal of neurointerventional surgery, neurintsurg-2014. 